\title{
Alterações eletrocardiográficas em pacientes com traumatismo da coluna cervical
}

\author{
Carlos Umberto Pereira ${ }^{1}$, Antonio Carlos Sobral Sousa ${ }^{2}$, Meyline Andrade
}

Lima $^{3}$, Pâmera Cristal Fontes Santos ${ }^{3}$, Egmond Alves Silva Santos ${ }^{4}$

Hospital Governador João Alves Filho, Aracaju, SE, Brasil

Trabalho realizado com auxílio de Bolsa de Iniciação Científica PIBIC/CNPq/UFS

\section{RESUMO}

O coração é inervado pelo sistema simpático oriundo da coluna cervical e pelo sistema parassimpático do bulbo. Em casos de traumatismo da coluna cervical, ocorre falha do controle simpático do coração, predominando o sistema vagal, visto que este não é lesado. Esse predomínio é manifestado clinicamente por bradicardia e hipotensão. O presente trabalho objetivou analisar os achados eletrocardiográficos em pacientes jovens, sem antecedentes de cardiopatia, vítimas de traumatismo da coluna cervical. Foram analisados 35 pacientes (31 do sexo masculino e 4 do feminino). A idade média dos pacientes foi de $30 \pm 8$ anos. Em $65 \%$ dos casos, encontravam-se tetraplégicos. Houve predomínio de lesão entre C5 e C6 em $48 \%$ dos casos. Os achados eletrocardiográficos apresentaram ritmo sinusal. A freqüência cardíaca média era de $74 \pm 16 \mathrm{bpm}$ e o eixo elétrico de $56 \pm 17^{\circ}$. Em $28,17 \%$ dos casos, o eletrocardiograma foi normal. Os achados anormais no eletrocardiograma foram: ondas T negativas na derivação V1 (17,18\%), bloqueio de ramo direito (36,46\%), distúrbios de repolarização precoce $(36,46 \%)$ e ondas $T$ amplas em V4 (9\%). A maioria desses achados é considerada fisiológica, não sendo eles tidos como predominantes em pacientes com traumatismo raquimedular cervical.

\section{PALAVRAS-CHAVE}

Alterações eletrocardiográficas. Traumatismo raquemedular cervical.

\section{ABSTRACT}

Electrocardiographic alterations in patients with cervical spine injury

The heart receives nervous by the deriving likeable system of the cervical column and parasimpatic system of the bulb. In cases of trauma of the cervical column, imperfection of the likeable control of the heart occurs, predominating the vagal system, since this is not injured. This predominance is revealed clinically by bradycardia and hypotension. The present work objectified to analyze the electrocardiographic findings in young patients, without antecedents of cardiopathy, victims of trauma of the cervical column. 35 patients, being 31 of masculine the sex and four feminine one had been analyzed. The average age of the patients was of $30 \pm 8$. In $65 \%$ of the cases they met in tetraplegy. It had predominance of injury between $\mathrm{C} 5$ and $\mathrm{C} 6$, in $48 \%$ of the cases. The electrocardiographic findings had presented sinusal rhythm. The average cardiac frequency was of $74 \pm 16 \mathrm{bpm}$ and the electric axle of $56 \pm 17^{\circ}$. In $28,17 \%$ of the cases the electrocardiography was normal. The abnormal findings in the electrocardiography had been, negative waves $T$ in the $V 1$ derivation in 17,18\%, blockade of right branch in $36,46 \%$, riots of precocious repolarization in $36,46 \%$ and ample waves $T$ in $V 4$ in $9 \%$ of the cases. The majority of these findings is considered physiological, not being considered as predominant in patients with cervical spine injury.

\section{KEY WORDS}

Cervical spine injury. Electrocardiographic alterations.

1 Professor adjunto Doutor do Departamento de Medicina da Universidade Federal de Sergipe. Neurocirurgião do Hospital João Alves Filho. Aracaju, SE.

2 Professor adjunto Doutor do Departamento de Medicina da Universidade Federal de Sergipe.

3 Doutoranda de medicina da Universidade Federal de Sergipe.

4 Médico residente de neurocirurgia do Conjunto Hospitalar do Mandaqui. São Paulo, SP. 


\section{Introdução}

O traumatismo raquimedular (TRM) é definido como uma lesão traumática aguda sobre a coluna vertebral e seu conteúdo nervoso, com graus variáveis de deficiência motora e/ou sensitiva, excluindo-se as doenças degenerativas crônicas e os procedimentos cirúrgicos ${ }^{16}$.

O presente trabalho refere-se ao estudo epidemiológico prospectivo das alterações eletrocardiográficas em pacientes com TRM cervical admitidos no setor de emergência do Hospital Governador João Alves Filho, em Aracaju, SE.

\section{Pacientes e métodos}

\section{Identificação dos pacientes}

Foram analisados pacientes de ambos sexos, com idade inferior a 40 anos e sem cardiopatia prévia, admitidos no setor de emergência do Hospital Governador João Alves Filho, apresentando traumatismo da coluna cervical, no período entre agosto e dezembro de 2004.

\section{Exame neurológico}

Foram submetidos a exame neurológico objetivando evidenciar alterações sensitivomotoras e compará-las com as alterações encontradas nos exames de imagens.

\section{Exames radiológicos}

Foram realizados exames de radiografia simples da coluna cervical nas incidências anteroposteriores (AP) e perfil, tomografia computadorizada e, quando possível, ressonância magnética da coluna cervical, a fim de confirmar a existência e o nível da lesão.

\section{Eletrocardiograma}

Os pacientes foram submetidos a exames eletrocardiográficos dentro das primeiras 24 horas do trauma, a fim de se observar possíveis e quaisquer alterações.

\section{Resultados}

Foram analisados 35 pacientes admitidos no Centro de Trauma do Hospital Governador João Alves Filho apresentando traumatismo da coluna cervical (31 do sexo masculino e 4 do feminino). A idade variou entre 19 e 36 anos (média de 31 anos).

Entre os mecanismos causadores do trauma, 17 pacientes foram vítimas de acidente de trânsito $(48,57 \%), 6$ de ferimento por arma de fogo $(17,14 \%)$, 6 de queda de altura $(17,14 \%), 4$ após mergulho em águas rasas $(11,42 \%)$ e 2 por ferimento por arma branca $(2,85 \%)$.

A maior parte de nossos pacientes encontravase tetraplégica $(65 \%), 15 \%$ não tinham alterações de sensibilidade ou motoras e $20 \%$ apresentavam alterações autonômicas.

Foi realizada radiografia da coluna cervical em incidência ântero-posterior e perfil e tomografia computadorizada da coluna cervical em todos os pacientes. A ressonância magnética foi realizada em 15 pacientes. Com relação à topografia das lesões, $92 \%$ dos pacientes apresentaram lesão entre C3-C7 e 8\% possuíam trauma em região occipto-C1-C2.

Todos os exames eletrocardiográficos apresentaram ritmo sinusal. A freqüência cardíaca média dos pacientes era de $74 \pm 16 \mathrm{bpm}$ e o eixo elétrico, de $56 \pm 17^{\circ}$. Em $28,17 \%$ dos casos, o eletrocardiograma não demonstrou alteração. Um total de $36,46 \%$ dos indivíduos apresentavam bloqueio de ramo direito; $36,46 \%$, distúrbios de repolarização precoce; $17,18 \%$, ondas $\mathrm{T}$ negativas na derivação $\mathrm{V} 1 ; 9 \%$, ondas $\mathrm{T}$ amplas em V4.

\section{Discussão}

A coluna vertebral consiste de 33 vértebras unidas por múltiplos ligamentos e interpostas por cartilagens. Existem 12 vértebras torácicas, 7 cervicais, 5 lombares, 5 sacrais e 1 coccígea. Ela funciona como um excelente mecanismo de proteção para a medula espinhal ${ }^{23}$.

Da medula espinhal, originam-se nervos que participam da inervação autonômica simpática do coração. A inervação parassimpática é feita pelo nervo vago que emerge do sulco lateral posterior do bulbo. O plexo cardíaco é composto por três nervos cardíacos cervicais do simpático (superior, médio e inferior), pelos dois nervos cardíacos cervicais do vago (superior e inferior), além dos nervos cardíacos torácicos do simpático e do vago. Embora o coração tenha sua posição torácica, ele recebe sua inervação predominantemente da região cervical, o que se explica por sua origem na região cervical do embrião ${ }^{19}$.

O TRM é definido como uma lesão aguda da coluna vertebral e seu conteúdo, com graus variáveis de deficiência motora e/ou sensitiva, excluindo-se as 
doenças degenerativas crônicas, os procedimentos cirúrgicos e os traumas neonatais ${ }^{16}$. $\mathrm{O}$ relato mais antigo de que se tem conhecimento acerca de um TRM é datado de entre 3.000 e 2.500 anos a.C., a partir de um papiro encontrado na grande pirâmide de Gizé ${ }^{36}$.

Apesar de não se encontrarem estatísticas disponíveis acerca dos TRM em nosso meio, pode-se avaliar sua incidência em importância a partir das referências da literatura mundial ${ }^{9}$. Admite-se que ocorram entre 11,5 e 60:1.000.000 casos a cada ano ${ }^{3,12}$, com estimativa média de 30 a 40 casos para os países industrializados ${ }^{15,37}$. Nos Estados Unidos, ocorrem 10.000 casos novos de TRM por ano ${ }^{37}$. Atualmente, é aceito que, dos pacientes que evoluem para óbito em razão de um trauma de qualquer natureza, em torno de 5\% apresentam TRM, seja este a causa direta da morte ou associação a traumatismos de outros órgãos e sistemas ${ }^{37}$.

A maior incidência de TRM ocorre no sexo masculino ${ }^{12}$, com variação de $2,4: 1$ até $8,5: 1$ a razão homem:mulher ${ }^{16}$. Quanto à idade, é observada maior incidência entre 16 e 25 anos, com novo pico após os 55 anos de idade. O primeiro pico é atribuído às características próprias de indivíduos jovens, que sofrem maior número de acidentes de trânsito, se envolvem mais facilmente em situações nas quais possam sofrer agressões físicas ou, principalmente, às práticas esportivas. No segundo, seria conseqüente às quedas associadas a alterações degenerativas prévias da coluna vertebral (em especial da cervical), muito comuns em pacientes idosos ${ }^{37}$. Considerando-se os TRM quanto à sua causa, na maioria dos estudos epidemiológicos realizados nos países desenvolvidos, é observado que os acidentes de trânsito são responsáveis por cerca de $50 \%$ deles, seguidos de quedas $(25 \%)$ e de acidentes esportivos em geral $(10 \%)$, prevalecendo nestes últimos os mergulhos em águas rasas ${ }^{28}$.

Visto que a inervação autônoma simpática do coração é proveniente da medula torácica de T1 a T5, lesões acima desse nível podem cursar com vários tipos de arritmias em conseqüência do aumento da atividade reflexa simpática devido à falta de controle pelos centros encefálicos superiores. Existe, no entanto, um predomínio vagal, porque este não é lesado, visto que se origina no bulbo raquidiano, manifestado por bradicardia e vasodilatação, resultando em hipotensão arterial ${ }^{24}$.

Freitas ${ }^{9}$, em 1990, observou, em seu estudo com 100 pacientes que sofreram TRM, a nítida prevalência dessa patologia no sexo masculino. Tal fato, observado na maior parte das lesões traumáticas de qualquer natureza, pode ser atribuído ao tipo de atividade desempenhada por esse sexo, mais exposto aos acidentes de trânsito, de trabalho e às práticas esportivas, assim como por seu envolvimento em agressões físicas. Isso também pode ser visto em nosso estudo, no qual as lesões medulares por trauma cervical também ocorreram, na maioria dos casos, em indivíduos do sexo masculino.

Com relação à idade, é observado um pico de incidência entre 16 e 25 anos, com novo pico após os 55 anos de idade ${ }^{14}$. Ocorrem mais comumente no verão, sobretudo devido a um incremento nos esportes e atividades aquáticas ${ }^{1}$. Além disso, é relatado que os acidentes de trânsito em geral e as agressões físicas são mais freqüentes nos fins de semana e nos meses de verão ${ }^{15}$

A maioria dos TRM é causada por acidentes de trânsito $(50 \%)$, seguidos por quedas $(25 \%)$ e acidentes esportivos em geral (10\%), sendo que, entre estes últimos, prevalecem os mergulhos em águas rasas ${ }^{28,32}$. São mais comuns nas vértebras cervicais médio-inferiores (C5-C6), seguidas da transição toracolombar ${ }^{33}$. A principal causa do TRM em nosso meio é o acidente de trânsito, o que ocorre na maioria dos centros urbanos devido ao desenvolvimento contínuo da tecnologia, com a criação de motores cada vez mais potentes. Ele atinge uma população predominantemente jovem, deixando-os tetraplégicos, na maioria dos casos, de lesões cervicais completas. Nos TRM cervicais, a localização mais comum da lesão medular foi entre C5 e C6. No exame neurológico desses pacientes, pode-se observar que entre $50 \%$ e $74 \%$ deles apresentam tetraplegia ou paraplegia ${ }^{7,8}$. Sua mortalidade é de aproximadamente $50 \%{ }^{15,16}$.

O TRM cervical pode afetar a inervação simpática do coração, havendo desconexão dos centros superiores e, conseqüentemente, predomínio parassimpático, visto que este se origina no bulbo raquidiano. O predomínio vagal manifesta-se por bradicardia e vasodilatação abaixo da lesão, com conseqüente hipotensão arterial ${ }^{24}$. Os fatores taquicardizantes como hipóxia, hipovolemia e hipertermia modificam pouco a freqüência cardíaca ${ }^{2}$. Tais sintomas são mais evidentes em lesões medulares cervicais completas e durante os primeiros dias após a lesão.

O TRM é com freqüência associado a uma variedade de anormalidades cardiovasculares, incluindo bradicardia, hipotensão arterial, arritmias supraventriculares e parada cardíaca. A bradicardia que progride para a assistolia é uma complicação freqüente do TRM cervical agudo ${ }^{4}$. Em lesões medulares cervicais completas, a bradicardia ocorre em $100 \%$ dos pacientes, enquanto a assistolia aparece em $15 \%{ }^{18}$. A bradicardia é limitada às primeiras três a cinco semanas pós-TRM cervical ${ }^{34}$. 
Em todos os casos de TRM, antes de se atribuir a bradicardia à instabilidade autonômica, a hipóxia deve ser excluída. Em pacientes quadriplégicos, esta pode ser resultado de disfunção diafragmática, presença de atelectasias, consolidações e depressão respiratória por sedação ou uso de analgésicos narcóticos. A aspiração traqueal pode causar ambas, hipóxia e estimulação vagal, resultando em bradicardia ${ }^{31}$.

Outros possíveis mecanismos das arritmias cardíacas nesses pacientes incluem trauma ou isquemia cerebral, lesão miocárdica e resposta vasovagal ao estresse emocional ${ }^{31}$. A estimulação do neocórtex, dos gânglios da base, do hipotálamo e do mesencéfalo produz uma variedade de arritmias supraventriculares e ventriculares, bem como uma alteração da pressão arterial através de mudanças na atividade simpática e/ou parassimpática ${ }^{21,27}$.

Existe uma relação direta entre a severidade de lesão medular e a incidência e a severidade dos problemas cardiovasculares ${ }^{25}$. Uma bradicardia persistente é muito mais comum em pacientes com lesões cervicais completas do que naqueles com lesões incompletas ou lesões toracolombares. Lehamann e cols. ${ }^{18}$, em seu estudo sobre as anormalidades cardiovasculares em pacientes com trauma medular, observaram que a bradicardia sinusal, a hipotensão, as arritmias supraventriculares e a assistolia foram significativamente mais comuns nos pacientes com lesão medular cervical severa quando estes foram comparados aos pacientes com TRM toracolombar.

Metha e cols. ${ }^{22}$, em 2001, descreveram o caso de um paciente com trauma medular cervical agudo que apresentou bradicardia (50 bpm) e hipotensão arterial (pressão arterial de 70 x $46 \mathrm{mmHg}$ ). O eletrocardiograma demonstrou bradicardia sinusal, bloqueio de ramo direito e aumento do intervalo QT. Greenhoot e cols. ${ }^{11}$, em experimentos utilizando cachorros submetidos a compressão da medula cervical, observaram o envolvimento de ambos os sistemas simpático e parassimpático produzindo sérios distúrbios do ritmo cardíaco, alterações do segmento ST e da onda T e elevações da pressão arterial.

Evans e cols. ${ }^{6}$ demonstraram experimentalmente que severas arritmias cardíacas e episódios de hipertensão arterial ocorriam após compressão da medula espinhal. De início, ocorria bradicardia sinusal ou pelo nodo atrioventricular, que era mediada pelo sistema nervoso autônomo parassimpático, visto que podia ser eliminada pelos bloqueadores colinérgicos. Diversas e severas arritmias que apareciam posteriormente foram atribuídas aos sistemas simpático e parassimpático, por necessitarem tanto de agentes bloqueadores simpáticos como parassimpáticos para serem eliminadas.
Sintomas crônicos da diminuição da inervação simpática são encontrados raramente; porém, episódios de bradicardia persistente têm sido descritos ${ }^{10,20}$. A hipotensão arterial não é adequadamente tratada com a infusão de fluidos, mas tem-se mostrado responsiva ao uso de vasoconstritores ${ }^{10,18}$. A prevalência de bradiarritmias é tanto maior quanto maior for a condição de hipotensão arterial ${ }^{20}$.

O método de tratamento da bradicardia devido ao trauma da medula espinhal depende de uma série de fatores. Eles incluem a idade e o estado geral do paciente, o tempo de aparecimento após o trauma, o nível da lesão medular e se a lesão é completa ou não. Também é muito importante considerar se o paciente é sintomático ou não durante os episódios ${ }^{31}$.

Como a bradicardia é devida a uma dominante e/ou uma excessiva atividade parassimpática, as drogas anticolinérgicas como a atropina e a propantelina podem ser eficazes nesses casos. Drogas simpaticomiméticas como a isoprenalina também podem ser eficientes, porém causam significativamente mais arritmias ${ }^{31}$. A implantação de marcapassos não prolonga a vida ${ }^{34}$; no entanto, deve ser utilizado em pacientes com bradiarritmia severa que não responde ao tratamento medicamentoso.

Imediatamente após uma lesão medular, produzse uma inibição de toda atividade nervosa abaixo da lesão, quadro conhecido como choque medular, que se manifesta clinicamente por flacidez muscular, arreflexia osteotendinosa, disfunção erétil, paralisia vesical e retal ${ }^{24}$. Uma vez passada a fase de choque medular, o sistema nervoso simpático começa a funcionar sem controle das funções superiores, de forma reflexa; com isso, os vasos sanguíneos adquirem tônus vascular por atividade reflexa simpática, que nunca chega a ser tão perfeito como em um individuo normal, o que determina flutuações na pressão arterial ${ }^{24}$.

A estimulação de receptores localizados em qualquer região abaixo do nível lesional e situados preferencialmente na região urogenital ${ }^{5}$ pode desencadear um reflexo autonômico simpático maciço, denominado hiperreflexia autonômica simpática (HRAS), que cursa com hipertensão arterial paroxística associada a bradicardia $^{26,29}$. Várias fontes de HRAS têm sido relatadas, sendo a distensão vesical a principal, seguida pela distensão retal ${ }^{13,26,35}$.

Esses estímulos sensoriais são transmitidos pelos feixes epinotalâmicos e cordões posteriores, trafegando em direção ao hipotálamo. Porém são bloqueados ao nível da lesão, determinando hiperatividade simpática generalizada ${ }^{17}$. Há súbita elevação da pressão arterial e espasmo arteriolar da pele e vísceras ao nível da lesão ${ }^{30}$. Com tal aumento da pressão arterial, barorreceptores no arco aórtico, nos corpos carotídeos 
e nos vasos encefálicos desencadeiam impulsos para o centro vasomotor, que responde originando dois arcos reflexos no tronco encefálico: 1) via núcleo motor do vago, estimulando receptores colinérgicos cardíacos e produzindo bradicardia; 2) inibitório descendente para as vias simpáticas na pele e na circulação esplâncnica que nunca atinge seu destino (exceto nas porções superiores), dada a presença de lesões medulares ${ }^{30}$.

Em nosso estudo, todos os pacientes com TRM cervical agudo apresentavam ritmo sinusal ao eletrocardiograma. Em $28,17 \%$ dos casos, o eletrocardiograma apresentou-se sem nenhuma alteração. Ondas T negativas na derivação V1 apareceram em 17,18\%, $36,46 \%$ apresentavam bloqueio de ramo direito, $36,46 \%$ distúrbio de repolarização precoce e em $9 \%$ pôde-se ver ondas $\mathrm{T}$ amplas em V4, que nos indicam uma vagotonia, esperada na maioria dos casos. A maior parte das alterações citadas pode ser encontrada em indivíduos normais, o que nos impede de rotulá-las como alterações presentes em indivíduos portadores de TRM cervical.

\section{Conclusão}

Apesar de traumatismos medulares cervicais serem muitas vezes relatados em associação a uma variedade de anormalidades cardiovasculares (incluindo bradicardia, hipotensão arterial, arritmias supraventriculares e parada cardíaca), grande parte das alterações encontradas em nossa casuística pode ser verificada em indivíduos normais, o que nos impede de rotulá-las como alterações conseqüentes à lesão medular cervical.

\section{Referências}

1. ALBRAND OW, CORKILL G: Broken necks from diving accidents. Am J Sports Med 4:107-10, 1976.

2. BAROISA, GSCHAEDLER R: Les troubles neurovégetatifs cardiovasculaires. In La Paraplégie chez l'adulte et chez l'enfant. Muury, París, 56-7, 1982.

3. CLIFTON GL: Spinal cord injury in the Houston-Galveston area. Tex Med 79:55-57, 1983.

4. DIXIT S: Bradycardia associated with high cervical spinal cord injury. Surg Neurol 43:514, 1995.

5. ERICKSON RP: Autonomic hyperrreflexia: pathophysiology and medical management. Arch Phys Med Rehabil 61:43140, 1980.

6. EVANS DE, KOBRINEAI, RIZZOLI HV: Cardiac arrhythmias accompanying acute compression of the spinal cord. J Neurosurg 52:52-9, 1980.
7. FINE PR, KUHLEMEIER KV, DE VIVO WJ, STOVER SL: Spinal cord injury: an epidemiology perspective. Paraplegia 17:237-50, 1979.

8. FRANKEL HL, HANCOCK DO, HYSLOP G, MELZAK J, MICHAELIS LS, UOGAR GH et al.: The value of postural reduction in the initial management of closed injuries of spine with paraplegia and tetraplegia. Paraplegia 7:179-92, 1969.

9. FREITAS PEP: Traumatismos raquimedulares agudos: estudo epidemiológico de 100 casos consecutivos. J Bras Neurocirurg 2:1-10, 1990.

10. GILGOFF I, DAVIDSON-WARD S, HOHN S: Cardiac pacemaker in high spinal cord injury. Arch Phys Med Rehabil 72:601-3, 1991

11. GREENHOOT JH, SHEIL FO'M, MAUCK HP: Experimental spinal cord injury. Arch Neurol 26:524-9, 1972.

12. GRIFFIN MR, O'FALLON WM, OPITZ JL, KURLAND LT: Mortality, survival and prevalence: traumatic spinal cord injury in Olmsted County, Minnesota, 1935-1981. J Chron Dis 38:643-53, 1895.

13. HALL PA, YOUNG JV: Autonomic hyperreflexia in spinal cord injured patients: trigger mechanism-dressing changes of pressure sores. J Trauma 23:1074-5, 1983.

14. KALSBEEK WD, MCLAURIN RL, HARRIS BSH, MILLER JD: The national head and spinal cord injury survey: major findings. J Neurosurg 53:519-31, 1980.

15. KÖNING W, FROWEIN RA, FIRSCHING R.: Epidemiology of spinal cord injury. In VIGOUROUX RP, HARRIS PH: Advances in Neurotraumatology - Thoracic and lumbar spine and spinal cord injuries. Springer-Verlag, Wien-New York, Vol 2, pp 1-15, 1987.

16. KRAUS JF, FRANTI CE, RIGGINS RS, RICHARDS D, BORHANI NO: Incidence of traumatic spinal cord lesions. J Chron Dis 28:471-92, 1975.

17. KURNICK NB: Autonomic hyperreflexia and its control in patients with spinal cord lesions. Ann Intern Med 44:67886, 1956.

18. LEHMAN K, LANE J, PIEPMEIR J, BATSFORD W: Cardiovascular abnormalities accompanying acute spinal cord injury in humans: incidence, time course and severity. J Am Coll Cardiol 10:46-52, 1987.

19. MACHADO ABM: Sistema nervoso autônomo: anatomia do simpático, parassimpático e dos plexos viscerais. In MACHADO ABM.: Neuroanatomia Funcional. Atheneu, Rio de Janeiro, Ed 2, pp 139-50, 1993.

20. MATHIAS CJ: Bradycardia and cardiac arrest during tracheal suction: mechanisms in quadriplegic patients. Eur $\mathrm{J}$ Intensive Care Med 2:147-56, 1976.

21. MAUCK HP, HOCHMAN CH: Central nervous system mechanisms mediating cardiac rate and rhythm. Am Heart J 74:96, 1967.

22. MEHTANJ, MEHTARN, KHAN IA: Electrocardiographic changes of acute cervical spinal cord trauma. Clin Cardiol 24:165, 2001.

23. NETTER FH: Nervous system. In The Ciba collection of medical illustrations. Vol 1, 1975.

24. PAYNO PB, BERTOL CL, ÁLVAREZ JM: Alteraciones cardiovasculares en los lesionados medulares cervicales. Rev Clín Española 188:24-7, 1991.

25. PEIPMEIER JM, LEHMANN KB, LANE JG: Cardiovascular instability following acute cervical spinal cord trauma. Cent Nerv Syst Trauma 2:153-60, 1985.

26. REDER JC, GISVOLD SE: Perioperative autonomic hyperreflexia in high spinal cord lesions: a case report. Acta Anaesthesiol Scand 30:672-3, 1986.

27. REIS DJ, OLIPHANT MC: Bradycardia and tachycardia following electrical stimulation of the amygdaloid region in monkey. J Neurophysiol 27:893, 1964. 
28. RIGGINS RS, KRAUS JF: The risk of neurological damage with fracture of the vertebrae. J Trauma 17:126-33, 1977.

29. SETTON ARF, PEREIRA CU, SANTOS JL: Hiperreflexia autonômica simpática: Revisão da literatura. J Bras Neurocirurg 6:49-54, 1995

30. SHEA JD, GIOFFRE R, CARRION H, SMALL MP: Autonomic hyperreflexia in spinal cord injury. South Med $J$ 66:869-72, 1973.

31. SILBERT PL, DAVIS MJE: Late asystole in high cervical spinal cord injury: Case report. Paraplegia 28:137-40, 1990.

32. SODERSTROM CA, BRUMBACK RJ: Early care of the patient with cervical spine injury. In LEVINE AM.: Acute spinal injury. Philadelphia, WB Saunders, vol. 17, cap 1, pp 3-13, 1980.

33. TATOR $\mathrm{CH}$, EDMONDS VE: Acute spinal cord injury: analysis of epidemiologic factors. Can J Surg 22:575622, 1979.

34. WINEINGER MA, BASFORD JR: Autonomic dysreflexia due to medication: misadventure in the use of an isometheptene combination to treat migraine. Arch Phys Med Rehabil 66:645-6, 1985.
35. WINSLOW EBJ, LESCH M, TALANO JV, MEYER PR: Spinal cord injuries associetad wilth cardiopulmonary complications. Spine 11:809-12, 1986.

36. YASHON D.: Epidemiology. In Spinal injury. Ed 2, Norewalk, Appleton-Century-Crofts, Cap. 2, pp 6-12, 1986.

37. YASHON D: History. In Spinal Injury. Ed 2, Norewalk, Appleton-Century-Crofts. Cap 1, pp 1-15, 1986.

Original recebido em dezembro de 2005

Aceito para publicação em abril de 2006

\section{Endereço para correspondência:}

Carlos Umberto Pereira

Av. Augusto Maynard, 245/404

49015-380 - Aracaju, SE

E-mail: umberto@infonet.com.br 